\title{
91. Singular Cauchy Problems for a Class of Weakly Hyperbolic Differential Operators
}

\author{
By Kanehisa TAKaSAKI \\ Department of Mathematics, University of Tokyo \\ (Communicated by Kôsaku Yosida, M. J. A., Oct. 12, 1981)
}

In these notes singular Cauchy problems of Hamada's type are studied in the category of holomorphic functions and hyperfunctions for a class of hyperbolic differential operators with non-involutive multiple characteristics. Integral representations of their solutions are given.

1. Introduction. Let $P\left(t, x, D_{t}, D_{x}\right)$ be a differential operator of order $m$ of the form

$$
P\left(t, x, D_{t}, D_{x}\right)=D_{t}^{m}+\sum_{i=1}^{m} A_{i}\left(t, x, D_{x}\right) D_{t}^{m-i},
$$

where $D_{t}=(1 / \sqrt{-1})(\partial / \partial t), D_{x}=(1 / \sqrt{-1})(\partial / \partial x)$ and $A_{i}\left(t, x, D_{x}\right)$ is a differential operator at most of order $i$, not containing $D_{t}$, whose coefficients are holomorphic functions defined in a neighborhood of $(t, x)$ $=(0,0)$ in $\boldsymbol{C} \times \boldsymbol{C}^{n}$.

We assume the following conditions :

(A-1) (Degeneracy of characteristic roots). There exists a nonnegative integer $q$ such that the principal symbol $P_{m}(t, x, \tau, \xi)$ of $P\left(t, x, D_{t}, D_{x}\right)$ is expressed in the form

$$
P_{m}(t, x, \tau, \xi)=\prod_{j=1}^{m}\left(\tau-t^{q} \lambda_{j}(\xi)\right),
$$

where $\lambda_{j}(\xi)(1 \leq j \leq m)$ are holomorphic functions defined in a conic open neighborhood $\Omega_{0}$ of $\xi_{0}=(1,0, \ldots, 0)$ in $C^{n}-0$ and homogeneous of degree 1 such that

$$
\lambda_{j}(\xi) \neq \lambda_{k}(\xi), \quad \text { if } j \neq k \text { and } \xi \in \Omega_{0} .
$$

(A-2) (Hyperbolicity). $\quad \lambda_{j}(\xi)(1 \leq j \leq m)$ are real if $\xi$ is real.

(A-3) (Levi condition). Let $A_{i, j}(t, x, \xi)$ be the homogeneous part of $A_{i}(t, x, \xi)$ of degree $j$ with respect to $\xi$ and let

$$
A_{i, j}(t, x, \xi)=\sum_{k=0}^{\infty} t^{k} A_{i, j, k}(x, \xi)
$$

be the Taylor expansion of $A_{i, j}(t, x, \xi)$ with respect to $t$. Then

$$
A_{i, j, k}(x, \xi)=0, \quad \text { if } k<(q+1) j-i .
$$

Alinhac [1], Amano [2], Amano-Nakamura [13], Nakamura-Uryu [6], Nakane [7], Taniguchi-Tozaki [10] and Yoshikawa [12] studied the Cauchy problem for weakly hyperbolic operators of the above type, and constructed parametrices, using a type of ordinary differential operators with polynomial coefficients which determine the principal 
parts of parametrices. (Nakamura-Uryu [6] and Amano-Nakamura [13] studied a more general case.) All of these authors, except Nakane [7], treated these subjects in the category of $C^{\infty}$ functions.

We deal with the singular Cauchy problem of Hamada's type $(\mathrm{CP})_{i} \quad\left\{\begin{array}{l}P\left(t, x, D_{t}, D_{x}\right) u_{i}(t, x, y, \xi)=0, \\ \left.D_{t}^{j} u_{i}\right|_{t=0}=\delta_{j, i}(\langle x-y, \xi\rangle+\sqrt{-1} 0)^{-n}\end{array} \quad\right.$ for $0 \leq j \leq m-1$, and its version in the complex domains $(\mathrm{CP})_{i}^{C} \quad\left\{\begin{array}{l}P\left(t, x, D_{t}, D_{x}\right) u_{i}(t, x, y, \xi)=0, \\ \left.D_{t}^{j} u_{i}\right|_{t=0}=\delta_{j, i}\langle x-y, \xi\rangle^{-n}\end{array}\right.$ for $0 \leq j \leq m-1$, for $0 \leq i \leq m-1$, where $\delta_{j, i}$ is Kronecker's delta, and show that the solution $u_{i}$ is obtained as an infinite series of Radon integrals (see (1.2)). Parametrices are obtained as integrals

where

$$
\int_{|\xi|=1} u_{i}(t, x, y, \xi) \omega(\xi) \quad(0 \leq i \leq m-1)
$$

$$
\omega(\xi)=\sum_{j=1}^{n}(-1)^{j-1} \xi_{j} d \xi_{1} \wedge \cdots \wedge d \xi_{j-1} \wedge d \xi_{j+1} \wedge \cdots \wedge d \xi_{n} .
$$

Our construction of solutions is similar to those of Yoshikawa [12] and Nakamura-Uryu [6], but we need much more delicate estimations. Our Radon integrals are modifications of those studied by Kataoka [4] and Aoki [3].

Before stating our main theorems we introduce the notations

$$
\begin{aligned}
& \psi_{j}(t, \xi)=(q+1)^{-1} \lambda_{j}(\xi) t^{q+1}, \\
& \varphi_{j}(t, x, y, \xi)=\langle x-y, \xi\rangle+\psi_{j}(t, \xi), \\
& r_{j}(t, \xi)=\max _{1 \leq k \leq m}\left|\psi_{j}\left(t, \xi_{1}^{-1} \xi\right)-\psi_{k}\left(t, \xi_{1}^{-1} \xi\right)\right|, \\
& d\left(t, \xi_{1}\right)=\left(|t|^{(q+1)}+\left|\xi_{1}\right|^{-1}\right)^{1 /(q+1)}, \\
& X=\left\{x \in C^{n} ;|x|<a\right\}, \\
& \Omega=\left\{\xi=\left(\xi_{1}, \xi^{\prime}\right) \in C^{n}-0 ;\left|\xi^{\prime}\right|<b\left|\xi_{1}\right|,\left|\arg \left(\xi_{1}\right)\right|<b\right\}, \\
& S_{\sigma}=\left\{t \in C-0 ;|\arg (\sigma t)|<(2 q+2)^{-1} \pi-\varepsilon\right\}, \\
& Z_{\sigma}=S_{\sigma} \times X \times \Omega, \quad Z=C \times X \times \Omega, \\
& D_{0}(r)=\{p \in C ; \operatorname{Im}(p)>r\}, \\
& D_{1}(d, r, R)=\bigcup_{-b<\theta<b}\left\{p \in C ; \operatorname{Im}\left(p e^{\sqrt{-1} \theta}\right)>r, d e^{R(|p|+r)}<1\right\},
\end{aligned}
$$

for positive constants $a, b, d, r, R$ and $\sigma= \pm 1,1 \leq j \leq m$.

Under Assumptions (A-1)-(A-3) we have

Theorem 1. For any sufficiently small positive constant $\varepsilon$, there exist positive constants $a, b, h$ and holomorphic functions $u_{\sigma, i, j}^{(\nu)}(\sigma= \pm 1$, $0 \leq i \leq m-1,1 \leq j \leq m, \nu \geq 0)$ defined in $Z$ such that the following hold:

(i ) A solution $u_{i}$ of $(\mathrm{CP})_{i}^{C}$ is obtained in the form

$$
u_{i}(t, x, y, \xi)=\sum_{j=1}^{m} u_{\sigma, i, j, R}\left(t, x, \xi ; \varphi_{j}(t, x, y, \xi)\right)+h_{\sigma, i, R}(t, x, y, \xi),
$$

for $\sigma= \pm 1$, where $u_{\sigma, i, j, R}$ is given by

$$
u_{\sigma, i, j, R}(t, x, \xi ; p)
$$




$$
=\sum_{\nu=0}^{\infty} \int_{(\nu+1) R}^{\infty} \exp \left(\sqrt{-1} \xi_{1}^{-1} p \rho\right) \xi_{1}^{-n} u_{\sigma, i, j}^{(\nu)}\left(t, x, \rho \xi_{1}^{-1} \xi\right) \rho^{n-1} d \rho,
$$

for any positive constant $R>h^{q+1}$, and $h_{\sigma, i, R}$ is a holomorphic function defined in a neighborhood of $(t, x, y, \xi)=\left(0,0,0, \xi_{0}\right)$ and homogeneous of degree $(-n)$ with respect to $\xi$.

(ii) $u_{o, i, j}^{(\nu)}$ satisfies (2.1)-(2.4).

(iii) The series (1.2) converges uniformly in every compact subset of the domain

$$
\begin{aligned}
& \left\{(t, x, \xi, p) \in Z_{\sigma} \times C ; d(t, R) h<1, \xi_{1}^{-1} p \in D_{0}(0)\right\} \\
& \cup \bigcap_{j=1}^{m}\left\{(t, x, \xi, p) \in Z \times C ; d(t, R) h<1, \xi_{1}^{-1} p \in D_{0}\left(r_{j}(t, \xi)\right)\right\},
\end{aligned}
$$

and hence it is a holomorphic function which is defined in this domain and homogeneous of degree $(-n)$ with respect to $(\xi, p)$. into

(iv) By deforming the integration path of the v-th term of (1.2)

$$
C_{\nu, R, \theta}=\{(\nu+1) R \exp (\sqrt{-1} s \theta) ; 0 \leq s \leq 1\} \cup\{(\nu+1) R s \exp (\sqrt{-1} \theta) ; 1 \leq s\}
$$
$(|\theta|<b), u_{\sigma, i, j, R}$ is continued to a holomorphic function defined in the domain

$$
\begin{aligned}
& \left\{(t, x, \xi, p) \in Z_{\sigma} \times C ; \xi_{1}^{-1} p \in D_{1}(d(t, R) h, 0, R)\right\} \\
& \cup \bigcap_{j=1}^{m}\left\{(t, x, \xi, p) \in Z \times C ; \xi_{1}^{-1} p \in D_{1}\left(d(t, R) h, r_{j}(t, \xi), R\right)\right\} .
\end{aligned}
$$

Theorem 2. The solution of $(\mathrm{CP})_{i}$ is given by the "boundary value hyperfunction" of (1.1), namely,

$$
\begin{aligned}
& u_{i}(t, x, y, \xi) \\
& \quad=\sum_{j=1}^{m} u_{\sigma, i, j, R}\left(t, x, \xi ; \varphi_{j}(t, x, y, \xi)+\sqrt{-1} 0\right)+h_{\sigma, i, R}(t, x, y, \xi) .
\end{aligned}
$$

The singularity support and the singularity spectrum of $u_{i}$, if we regard $\xi$ as a parameter, are estimated as follows :

$$
\begin{aligned}
& \text { sing. supp. }\left(u_{i}\right) \subset \bigcup_{j=1}^{m}\left\{\varphi_{j}=0\right\}, \\
& \text { S.S. }\left(u_{i}\right) \subset \bigcup_{j=1}^{m}\left\{\left(t, x, y ; \sqrt{-1} d \varphi_{j}(t, x, y, \xi) \infty\right) ; \varphi_{j}=0\right\} .
\end{aligned}
$$

(As for the terminologies of hyperfunctions and singularity spectra, we refer to Sato-Kawai-Kashiwara [9].)

Remark 1. As Amano [2] and Amano-Nakamura [13] pointed out, our method for the construction of solutions will be effective in the analysis of the "branching of singularities" at multiple characteristic points. Alinhac [1], Nakane [7] and Taniguchi-Tozaki [10] carried out the analysis in the case $m=2$, using special functions of the hypergeometric or confluent hypergeometric type.

Remark 2. (1.4) implies that the singularities of solutions are concentrated on the union of bicharacteristic strips associated with $\tau-t^{q} \lambda_{j}(1 \leq j \leq m)$ which pass $(t, x, y, \xi)=\left(0,0,0, \xi_{0}\right)$. This result is entirely different from those in the case of involutive multiple charac- 
teristics. (See, for example, Kawai-Nakamura [5].)

2. Outline of the proof. We choose $u_{o, i, j}^{(\nu)}(t, x, \xi)$ to be "semihomogeneous" of degree $(-i-\nu) /(q+1)$, namely, for $c \in C-0$,

$$
u_{\sigma, i, j}^{(\nu)}\left(c^{-1} t, x, c^{q+1} \xi\right)=c^{-i-\nu} u_{\sigma, i, j}^{(\nu)}(t, x, \xi)
$$

holds. Then, at least formally, we can reduce the problem to the "transport equation"

$$
\begin{aligned}
&\left(D_{t}^{m}+\sum_{k=1}^{m} A_{k}^{(0)}(t, x, \xi) D_{t}^{m-k}\right)(\left.\exp \left(\sqrt{-1} \psi_{j}(t, \xi)\right) u_{\sigma, i, j}^{(\nu)}\right) \\
&=-\sum_{k=1}^{m} \sum_{\substack{\alpha, \lambda, \alpha \geq 0, \nu=\kappa+\lambda+\alpha+1)|\alpha|,}} \frac{1}{\alpha \neq \lambda}\left(\partial_{\xi}^{\alpha} A_{k}^{(\kappa)}\right) D_{x}^{\alpha} D_{t}^{m-k} \\
& \times\left(\exp \left(\sqrt{-1} \psi_{j}(t, \xi)\right) u_{\sigma, i, j}^{(\lambda)}\right),
\end{aligned}
$$

with the "initial condition"

$$
\left.\sum_{j=1}^{m} D_{t}^{k}\left(\exp \left(\sqrt{-1} \psi_{j}\right) u_{\sigma, i, j}^{(\nu)}\right)\right|_{t=0}=\delta_{k, i} \delta_{\nu, 0}(\sqrt{-1})^{-n} /(n-1) !,
$$

for $0 \leq k \leq m-1,1 \leq k \leq m, \nu \geq 0$, where $\partial_{\xi}^{\alpha}=(\partial / \partial \xi)^{\alpha}$, and $A_{\sigma, i, j}^{(\nu)}(t, x, \xi)$ is defined by

$$
A_{i}^{(\nu)}(t, x, \xi)=\sum_{\substack{k \geq 0,0 \leq j \leq i \\ \nu=k-(q+1) j+i}} t^{k} A_{i, j, k}(x, \xi)
$$

Actually we can show that the proof is reduced to the construction of solutions of (2.2) and (2.3) which satisfy the "growth condition"

$$
\begin{aligned}
\left|D_{t}^{k} u_{\sigma, i, j}^{(\nu)}\right| \leq & C h^{\nu}\left|\xi_{1}\right|^{k-(q+1)-1 i} d\left(t, \xi_{1}\right)^{k q} d\left(t,(\nu+1)^{-1} \xi_{1}\right)^{\nu} \\
& \times \begin{cases}d\left(t \xi_{1}^{1 /(q+1)}, 1\right)^{\mu_{j}(x, \xi)}, & \text { if }(t, x, \xi) \in Z_{\sigma}, \\
d\left(t \xi_{1}^{1 /(q+1)}, 1\right)^{M(x, \xi)} e^{r_{j}(t, \xi)|\xi 1|}, & \text { if }(t, x, \xi) \in Z,\end{cases}
\end{aligned}
$$

for $0 \leq k \leq m$ and $\nu \geq 0$, where we set

$$
\begin{aligned}
\pi_{j}(x, \xi)= & -\sum_{i=1}^{m}\left\{(q / 2)(m-i+1)(m-i) A_{i-1, i-1, q(i-1)}(x, \xi)\right. \\
& \left.+\sqrt{-1} A_{i, i-1, q(i-1)-1}(x, \xi)\right\} \\
& \times \lambda_{j}(\xi)^{m-i} \prod_{k=1, k \neq j}^{m}\left(\lambda_{j}(\xi)-\lambda_{k}(\xi)\right)^{-1}, \\
\mu_{j}(x, \xi)= & \operatorname{Re}\left(\pi_{j}(x, \xi)\right), \quad M(x, \xi)=\max _{1 \leq j \leq m} \mu_{j}(x, \xi) .
\end{aligned}
$$

Since $\psi_{j}(t, \xi)$ and $A_{i}^{(\nu)}(t, x, \xi)$ are semi-homogeneous of degree 0 and $(i-\nu) /(q+1)$ respectively, (2.2)-(2.4) are compatible with the semihomogeneity. Namely, if $u_{\sigma, i, j}^{(\nu)}(t, x, \xi)(\sigma= \pm 1,1 \leq j \leq m, 0 \leq i \leq m-1$, $\nu \geq 0$ ) satisfy (2.2)-(2.4) with the constraint $\xi_{1}=1$ (which we abbreviate to $\left.\left.(2.2)\right|_{\xi_{1}=1},-\left.(2.4)\right|_{\xi_{1}=1}\right)$, and if we set

$$
u_{\sigma, i, j}^{(\nu)}(t, x, \xi)=\xi_{1}^{(-i-\nu) /(q+1)} u_{\sigma, i, j}^{(\nu)}\left(t \xi_{\nu}^{1 /(q+1)}, x, \xi_{1}^{-1} \xi^{\prime}\right),
$$

then they are solutions of (2.2)-(2.4). Thus essentially we have only to consider the case $\xi_{1}=1$.

We construct $u_{\sigma, i, j}^{(\nu)}$ by the induction on $\nu$.

For $\nu=0$, (2.2) $\left.\right|_{\xi_{1}=1}$ is a homogeneous ordinary differential equation with polynomial coefficients with respect to $t$, and has an irregular singular point of Poincaré's rank $(q+1)$ at $t=\infty$. It has formal solutions of the form 


$$
v_{j}=\hat{v}_{j} t^{\pi_{j}\left(x, 1, \xi^{\prime}\right)} \exp \left(\sqrt{-1} \psi_{j}\left(t, 1, \xi^{\prime}\right)\right) \quad \text { for } 1 \leq j \leq m,
$$

where $\hat{v}_{j}$ is a formal power series in $t^{-(q+1)}$ whose coefficients are holomorphic functions in $\left(x, \xi^{\prime}\right)$. By a version of the "asymptotic existence theorem" (Wasow [11, Theorem 12.3]), there exists, for each $j$, a holomorphic solution (not formal) of (2.2) $\left.\right|_{\xi_{1}=1}$ whose asymptotic expansion in the sector $S_{\sigma}$ coincides with $v_{j}$. Using these solutions we get solutions of (2.2)-(2.4) for $\nu=0$.

For $\nu \neq 0$, we construct solutions by the "method of the variation of constants", using the same integration paths as those which appeared in Nishimoto [8]. Through some delicate estimations we can show that these solutions actually satisfy the former half of (2.4).

To show the latter half of (2.4), we choose a suitable family of finitely many sectors which cover the whole plane $C$. Then we repeat similar arguments to express $u_{\sigma, i, k}^{(\nu)}$, for each sector $S$ of this family, in such a manner that

$$
u_{\sigma, i, k}^{(\nu)}=\sum_{j=1}^{m} \exp \left(\sqrt{-1}\left(\psi_{j}-\psi_{k}\right)\right) u_{\sigma, i, k ; S, j}^{(\nu)}
$$

holds, where $u_{\sigma, i, k ; S, j}^{(\nu)}$ satisfies the transport equations and the former half of (2.4) in $S$, instead of $S_{\sigma}$. This implies the latter half of (2.4).

The last argument is indispensable, because "Stokes' phenomena" (Wasow $[11, \S 15])$ may occur and the factor $\exp \left(\sqrt{-1}\left(\psi_{j}-\psi_{k}\right)\right)(j \neq k)$ may break the validity of the former half of (2.4) in the sector $S\left(\neq S_{\sigma}\right)$. This is the reason why we need the latter half of (2.4). This relates to the "branching of singularities". (See Remark 1.)

The detailed proof will appear elsewhere.

\section{References}

[1] S. Alinhac: Indiana Univ. Math. J., 27, 1027-1037 (1978).

[2] K. Amano: Proc. Japan Acad., 56A, 206-209 (1980).

[3] T. Aoki: Invertibility for microdifferential operators of infinite order (to appear in Publ. RIMS., Kyoto Univ.).

[4] K. Kataoka: On the theory of Radon transformations of hyperfunctions (to appear in J. Fac. Sci. Univ. Tokyo).

[5] T. Kawai and G. Nakamura: Publ. RIMS., Kyoto Univ., 14, 415-439 (1978).

[6] G. Nakamura and H. Uryu: Comm. in P. D. E., 5, 837-896 (1980).

[7] S. Nakane: Propagation of singularities and uniqueness in the Cauchy problem at a class of doubly characteristic points (to appear in Comm. in P. D. E.).

[8] T. Nishimoto: Kôdai Math. Sem. Rep., 18, 61-84 (1966).

[9] M. Sato, T. Kawai, and M. Kashiwara: Lect. Notes in Math., vol. 287, Springer (1973).

[10] K. Taniguchi and Y. Tozaki: Math. Japonica, 25, 279-300 (1980).

[11] W. Wasow: Asymptotic expansions for ordinary differential equations. Interscience (1965).

[12] A. Yoshikawa: Hokkaido Math. J., 6, 313-344 (1977).

[13] K. Amano and G. Nakamura: Proc. Japan Acad., 57A, 164-167 (1981). 